\title{
HIF prolyl hydroxylase inhibition protects skeletal muscle from eccentric contraction- induced injury
}

Andrew N. Billin ${ }^{1 \dagger}$, Samuel E. Honeycutt ${ }^{1 \dagger}$, Alan V. McDougal', Jaclyn P. Kerr ${ }^{1}$, Zhe Chen ${ }^{1}$, Johannes M. Freudenberg ${ }^{3}$, Deepak K. Rajpal ${ }^{3}$, Guizhen Luo ${ }^{1}$, Henning Fritz Kramer ${ }^{1}$, Robert S. Geske ${ }^{3}$, Frank Fang ${ }^{4}$, Bert Yao ${ }^{2}$, Richard V. Clark', John Lepore ${ }^{2}$, Alex Cobitz ${ }^{2}$, Ram Miller ${ }^{1}$, Kazunori Nosaka ${ }^{5}$, Aaron C. Hinken ${ }^{1}$ and Alan J. Russell ${ }^{{ }^{*}}$ (D)

\begin{abstract}
Background: In muscular dystrophy and old age, skeletal muscle repair is compromised leading to fibrosis and fatty tissue accumulation. Therefore, therapies that protect skeletal muscle or enhance repair would be valuable medical treatments. Hypoxia-inducible factors (HIFs) regulate gene transcription under conditions of low oxygen, and HIF target genes EPO and VEGF have been associated with muscle protection and repair. We tested the importance of HIF activation following skeletal muscle injury, in both a murine model and human volunteers, using prolyl hydroxylase inhibitors that stabilize and activate HIF.

Methods: Using a mouse eccentric limb injury model, we characterized the protective effects of prolyl hydroxylase inhibitor, GSK1120360A. We then extended these studies to examine the impact of EPO modulation and infiltrating immune cell populations on muscle protection. Finally, we extended this study with an experimental medicine approach using eccentric arm exercise in untrained volunteers to measure the muscle-protective effects of a clinical prolyl hydroxylase inhibitor, daprodustat.

Results: GSK1120360A dramatically prevented functional deficits and histological damage, while accelerating recovery after eccentric limb injury in mice. Surprisingly, this effect was independent of EPO, but required myeloid HIF1a-mediated iNOS activity. Treatment of healthy human volunteers with high-dose daprodustat reduced accumulation of circulating damage markers following eccentric arm exercise, although we did not observe any diminution of functional deficits with compound treatment.

Conclusion: The results of these experiments highlight a novel skeletal muscle protective effect of prolyl hydroxylase inhibition via HIF-mediated expression of iNOS in macrophages. Partial recapitulation of these findings in healthy volunteers suggests elements of consistent pharmacology compared to responses in mice although there are clear differences between these two systems.
\end{abstract}

Keywords: Eccentric injury, HIF activation, Skeletal muscle, Prolyl hydroxylase, Protection

\footnotetext{
* Correspondence: arussell@edgewisetx.com

${ }^{\dagger}$ Andrew N. Billin and Samuel E. Honeycutt contributed equally to this work.

${ }^{1}$ Muscle Metabolism Discovery Performance Unit, GlaxoSmithKline, King of

Prussia, PA, USA

Full list of author information is available at the end of the article
}

(c) The Author(s). 2018 Open Access This article is distributed under the terms of the Creative Commons Attribution 4.0 International License (http://creativecommons.org/licenses/by/4.0/), which permits unrestricted use, distribution, and reproduction in any medium, provided you give appropriate credit to the original author(s) and the source, provide a link to the Creative Commons license, and indicate if changes were made. The Creative Commons Public Domain Dedication waiver (http://creativecommons.org/publicdomain/zero/1.0/) applies to the data made available in this article, unless otherwise stated. 


\section{Introduction}

Under conditions of trauma or extreme physical exercise, skeletal muscle damage occurs and sets off an orchestrated series of events to replace and repair damaged tissue [1]. The dynamics of this process can be disrupted by chronic inflammation and age, resulting in inefficient replacement of muscle and the accumulation of connective tissue and fat $[2,3]$.

The transcription factor hypoxia-induced factor $1 \alpha$ $(\mathrm{HIF} 1 \alpha)$ is a primary regulator of the homeostatic response to hypoxia [2], and several lines of evidence point to the importance of hypoxic response to skeletal muscle repair. Both HIF1 $\alpha$ and the HIF-target gene, VEGF, are elevated by strenuous exercise $[4,5]$, and VEGF stimulates muscle angiogenesis and myoblast proliferation [6]. Another HIF-target gene, erythropoietin (EPO), also stimulates myoblast proliferation and muscle recovery after injury. One of the primary mediators of HIF $1 \alpha$ activity in muscle is local oxygen concentration, which can drop significantly during exercise or injury [7, 8]. Under normoxic conditions, oxygen-regulated HIF proteins (primarily HIF $1 \alpha$, HIF $2 \alpha$ ) are hydroxylated and targeted for proteasomal degradation by HIF-prolyl 4-hydroxylases (PHD1,2,3) which require molecular $\mathrm{O}_{2}$ for activity. Under low oxygen tension, PHD activity is reduced, stabilizing HIF and allowing transcription of target genes [9]. Recently, small molecule inhibitors of PHD proteins have been identified that stabilize HIF in vivo [10]. We took advantage of these pharmacologic tools to test the hypothesis that acute HIF activation would protect against muscle damage induced by lengthening (eccentric) muscle contractions and accelerate muscle repair in both mice and humans.

\section{Results}

Skeletal muscle is subject to dramatic changes in length and strain during contraction. One of the transcription factors involved in initiating the tissue response to acute exercise was recently shown to be HIF1 $\alpha$ [11]. We asked whether inhibiting PHD enzymes would be beneficial for muscle repair after injury. Using eccentric, lengthening contractions to cause acute muscle damage with subsequent inflammation and tissue remodeling [12] via a non-invasive footplate system in the gastrocnemius muscle of mice [13], muscle force was decreased on average by $30-40 \%$, but fully recovered to the initial force after 30 days. In contrast, daily dosage of the PHD inhibitor GSK1120360A (GSK360) [14], starting 10 min after

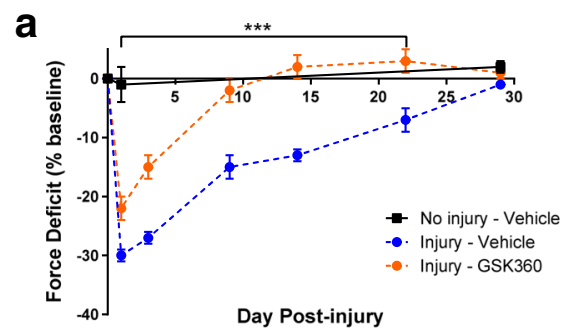

C

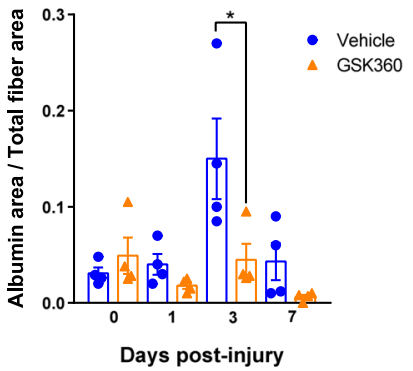

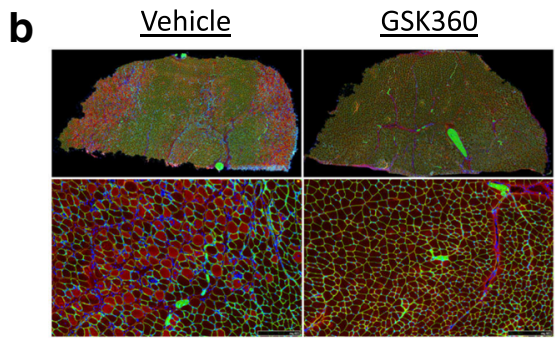

d

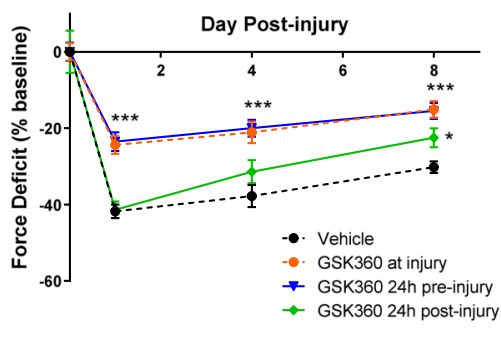

Fig. 1 Prolyl hydroxylase inhibitors protect skeletal muscle from exercise-induced injury. a Maximal limb force in mice before and after eccentric muscle exercise. Mice were treated with vehicle or GSK360 daily, starting 10 min after eccentric exercise. Limb force was measured for 28 days. Uninjured limb force included as a control. Data are normalized to pre-exercise force $(n=8)$. b Representative images of immunohistochemical staining for albumin (red), laminin (green), and nuclei (blue) in gastrocnemius muscle 3 days after eccentric exercise ( $n=4 /$ group). Upper panels: low power images of full cross section of gastrocnemius. Lower panels: higher magnifications of the rectangular areas above (scale bars= 250 MM). Arrows: albumin-positive myofibers. Asterisks: albumin-negative fibers. c Quantification of albumin-positive fibers measured as a proportion of total fibers ( $n=4$ /group). $\mathbf{d}$ Maximal limb force in mice before and after eccentric muscle damage. Mice were treated daily with vehicle or GSK360 $24 \mathrm{~h}$ before, at the time of injury, or $24 \mathrm{~h}$ after eccentric exercise. Limb force was measures for 8 days $(n=8)$. Data shown as the mean \pm SEM. Two-way ANOVA followed by Holm-Sidak's test corrected for multiple comparisons; ${ }^{*} P<0.05,{ }^{* *} P<0.01$, ${ }^{* * *} P<0.001$ versus vehicle-dosed cohort (unless otherwise indicated) 
injury, provided a significant protective effect at $24 \mathrm{~h}$ and full restoration of muscle force 9 days after damage (Fig. 1a, Additional file 1: Figure S1a). Furthermore, we found that fluorescent albumin incorporation was decreased by $70 \%$ 3 days post-injury with GSK360 treatment (Fig. 1b, c), indicating PHD inhibition also resulted in histological evidence of muscle protection.

As we saw a biphasic GSK360 effect on force recovery, we asked whether removing the initial protection from injury would still result in accelerated recovery. Instead of dosing at the time of injury, we delayed the initial dose of GSK360 until $24 \mathrm{~h}$ post-eccentric damage. Under these conditions, we found that recovery was still accelerated compared to control animals (Fig. 1d; GSK360 $24 \mathrm{~h}$ post-injury), indicating that activation of hypoxic signaling both decreased initial injury and accelerated muscle recovery.

Dosing with GSK360 has been shown to induce EPO production in vivo [14]. As EPO has been previously linked to improved muscle repair after injury [15], we tested whether the elevation of EPO by GSK360 was responsible for its protective effects. At the maximal effective dose $(10 \mathrm{mg} / \mathrm{kg})$ of GSK360, we found elevations in circulating EPO, but not VEGF (Fig. 2a, b). We then treated mice daily with recombinant EPO, with or without an EPO neutralizing antibody, beginning 2 days prior to eccentric injury to elucidate the effects of GSK360-induced EPO production on muscle recovery. Recombinant EPO was protective to muscle if administered before injury (Fig. 2c). However, pre-dosing with either an EPO neutralizing antibody, or a soluble EPO receptor decoy [16] prior to GSK360 treatment did not alter GSK360's protective muscle effects (Fig. 2d, e), while lowering Hct and RBC counts (Additional file 1: Figure S1b, c), suggesting that the protection afforded by GSK360 was not dependent on EPO.

That EPO was not required for muscle protection with GSK360 indicated another mechanism through which PHD inhibition might affect muscle injury, possibly via HIF $1 \alpha$ upregulation in muscle itself. HIF $1 \alpha$ protein was transiently elevated in compound treated muscle after injury (Fig. 2f), so we surveyed the early transcriptional effects of GSK360 in damaged muscle. Unexpectedly, we found no significant transcriptional changes specific to treatment in muscle (Additional file 2: Figure S2a, Additional file 3: Table S1) and that compound concentration in muscle was low relative to other organs (Additional file 2: Figure S2b), together suggesting another a

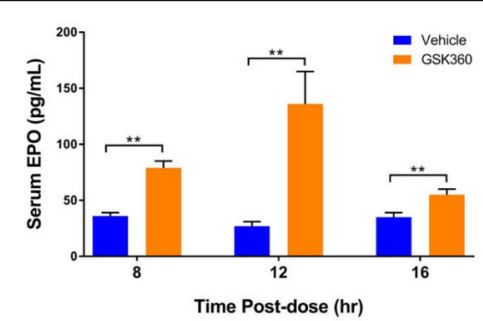

C

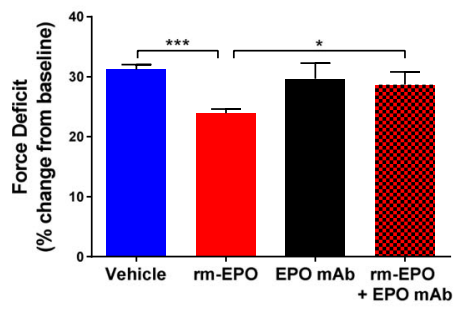

e

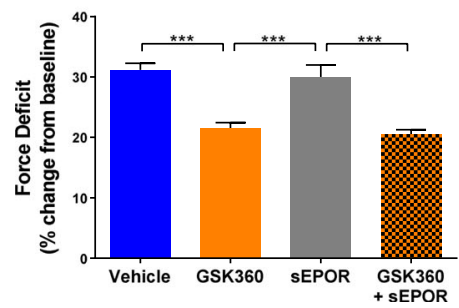

b

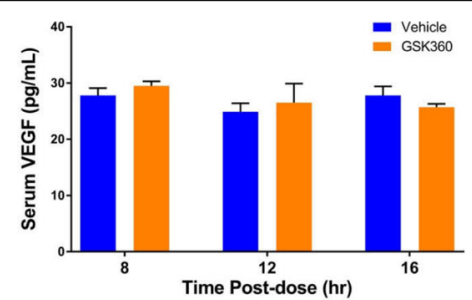

d

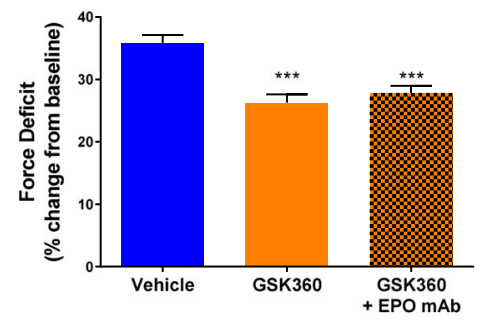

f

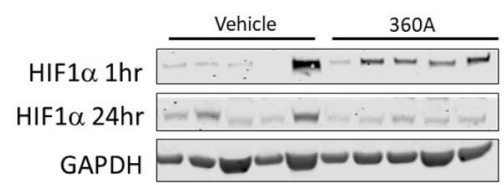

Fig. 2 Prolyl hydroxylase inhibitors exert their protective effects independently of EPO. a Serum EPO concentration and $\mathbf{b}$ Serum VEGF concentration after oral administration of $10 \mathrm{mg} / \mathrm{kg} \mathrm{GSK360} \mathrm{in} \mathrm{mice}(n=8)$. c Force deficit $24 \mathrm{~h}$ after eccentric injury. Mice were treated daily with $r E P O$, with and without an EPO neutralizing antibody $(n=8)$. d Force deficit $24 \mathrm{~h}$ after eccentric injury of mice co-treated with EPO neutralizing antibody and GSK360 $(n=8)$. e Force deficit $24 \mathrm{~h}$ after eccentric injury of mice co-treated with soluble EPO receptor and GSK360 $(n=8)$. $\mathbf{f}$ Western blot of HIF1a protein levels from muscle lysates $1 \mathrm{~h}$ and $24 \mathrm{~h}$ after limb injury. GAPDH used as a loading control 
tissue compartment was potentially responsible for the effects on muscle repair.

One possible effector that may play a role in the blunting of damage and acceleration of recovery would be the immune response following muscle damage. Cells of the myeloid lineage dominate muscle infiltrates after damage [17], and myeloid Hif1a knockout (HIF1 $\alpha$ KO) mice exhibit delayed recovery after muscle trauma [8] although this finding has been recently disputed [18]. Using myeloid HIF1 $\alpha \mathrm{KO}$ mice, we asked whether altering hypoxic signaling in immune cells would affect recovery from muscle injury. Using the same eccentric damage protocol as before, we found that GSK360 was not protective in either myeloid HIF1 $\alpha$ KO homo- or heterozygous mice (Fig. 3a), implicating HIF activation in myeloid cells in the muscle-protective response to GSK360.

Early myeloid infiltration into injured muscle is dominated by neutrophils and macrophages, with macrophages increasing in number and activity by $24 \mathrm{~h}$ after acute injury [19] (Additional file 2: Figure S2c). As macrophages play an important role in promoting skeletal muscle protection and repair [20], we asked whether HIF-responsive macrophages, rather than neutrophils, drove the primary GSK360 response in the muscle. To do so, we cultured bone marrow-derived macrophages (BMDMs) from both myeloid HIF1 $\alpha$ KO and myeloid HIF1 $\alpha$ expressing mice. The plasma membranes of these BMDM were fluorescently labeled with PKH67-green and injected intramuscularly into the gastrocnemius of myeloid HIF $1 \alpha \mathrm{KO}$ mice immediately following eccentric injury. Adoptive transfer of HIF $1 \alpha$ expressing macrophages re-established the protective effect of GSK360 seen in wild-type mice (c.f. Fig. 1), in contrast to those muscles that received HIF1 $\alpha$-deficient BMDM (Fig. 3b). FACS analysis of the myeloid cell population recovered from the gastrocnemius muscles $24 \mathrm{~h}$ following injury confirmed that most of the PKH67-green cells were positive for the macrophage marker, $\mathrm{CD} 11 \mathrm{~b}^{+}$ (Fig. 3c). We conclude that GSK360's protective effects are mediated through HIF activation and signaling in macrophages.

Macrophages are highly responsive to hypoxic signaling, with HIF1 $\alpha$ regulating a number of genes that may contribute to muscle protection including TNF $\alpha$, IL-1 $\beta$, iNOS, and VEGF [21]. Our primary focus was on iNOS

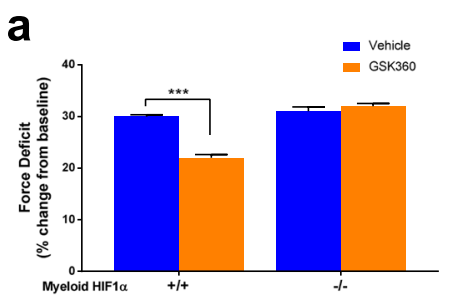

C
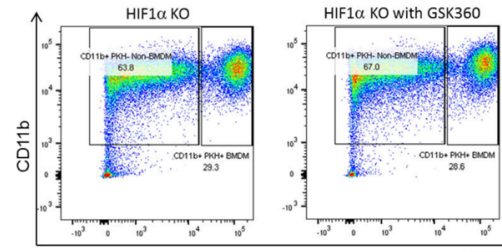

b

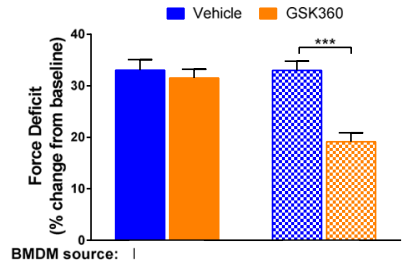

HIF1a. WT
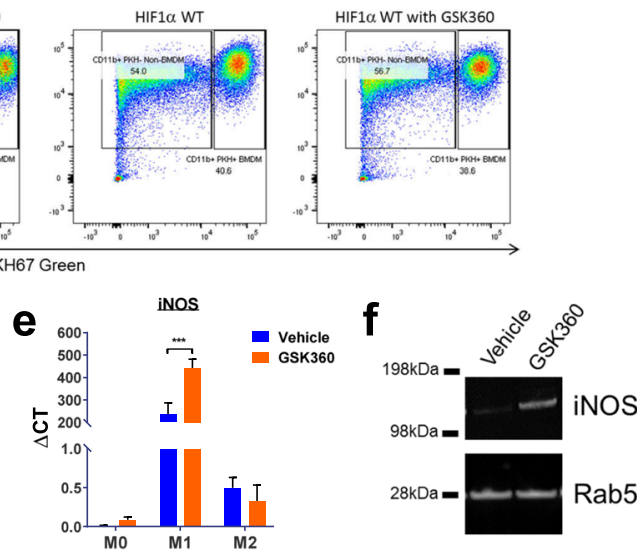

Fig. 3 Prolyl hydroxylase inhibitors exert their protective effects via macrophage HIF1a and iNOS. a Force deficit $24 \mathrm{~h}$ after eccentric injury in indicated strains of myeloid HIF1a KO mice administered vehicle or GSK360 $(n=8)$. b Force deficit $24 \mathrm{~h}$ after eccentric injury in myeloid HIF1a KO mice with exogenous BMDM (either HIF1a KO or WT) injected intramuscularly following eccentric injury and treated with vehicle or GSK360 $(n=8)$. c FACS analysis of PKH67-green labeled myeloid populations recovered from injured muscles $24 \mathrm{~h}$ after injection and treatment. $\mathbf{d}$ Force deficit $24 \mathrm{~h}$ after eccentric injury in WT mice treated with vehicle, iNOS inhibitor 1400 W and/or GSK360. Right side; force deficit 24 h after eccentric injury in iNOS KO mice administered vehicle or GSK360 ( $n=8)$. e iNOS gene expression measured by qPCR. Bone marrow-derived macrophages (BMDM) were cultured and polarized to promote classical M1 or M2 activation or left inactivated (M0) and treated with vehicle or GSK360 for 24 h. f iNOS protein expression in M1-polarized macrophages treated with GSK360 for 24 h before protein analysis by Western blot. Rab5 was used as a loading control. All data in the figure are shown as the mean \pm SEM. Two-way ANOVA followed by Holm-Sidak's test corrected for multiple comparisons; ${ }^{*} P<0.05,{ }^{* *} P<0.01,{ }^{* *} P<0.001$ versus the vehicle dosed cohort (unless otherwise indicated) 
as both nitric oxide [22] and iNOS itself [23] are regulators of myogenesis and muscle repair. Furthermore, they are highly expressed by classically activated M1 macrophages found to be present within the first $24 \mathrm{~h}$ following muscle injury [24], fitting the timeframe in which GSK360 appeared to be working. We first manipulated $\mathrm{NO}$ activity with pharmacological tools, co-treating mice with both GSK360 and $1400 \mathrm{~W}$, an iNOS inhibitor [25]. The addition of $1400 \mathrm{~W}$ blunted the GSK360 effect on muscle injury (Fig. 3d). We also measured force production after eccentric contractions in an iNOS KO mouse [26]; similarly to inhibiting iNOS, GSK360 failed to protect muscle after eccentric injury (Fig. 3d). Treating BMDM in culture with GSK360 resulted in increased expression of HIF1 $\alpha$ only in classically activated M1 macrophages (Additional file 2: Figure S2d), along with a dramatic increase in iNOS expression (Fig. 3e, f). Together, these data point to PHI mediating a protective effect in muscle via activation of HIF $1 \alpha$ in macrophages and the subsequent upregulation of iNOS.

In humans, performing unaccustomed eccentric exercise also leads to tissue damage, functional deficits, and muscle pain often referred to as delayed onset muscle soreness [27]. We took advantage of this to test the ability of a clinical stage PHI, daprodustat [28], to reduce muscle damage in healthy volunteers subjected to eccentric exercise of the elbow flexors in a double-blind placebo-controlled study. Daprodustat is structurally related to GSK360 and in preclinical studies also protected mice from functional deficits after eccentric injury (Additional file 4: Figure S3a). Subjects performed 30-60 contractions of their non-dominant arm to induce more than $40 \%$ strength loss immediately post-exercise and then were randomized to receive a total of five doses of either placebo or daprodustat (Fig. 4a). Arm function was measured as maximum voluntary contraction (MVC) force generated at a fixed elbow joint angle of $90^{\circ}$ and was recorded $10 \mathrm{~min}$ pre-exercise and $5 \mathrm{~min}, 30 \mathrm{~min}$, $24 \mathrm{~h}, 48 \mathrm{~h}$, and $72 \mathrm{~h}$ post-exercise. We also measured range of motion and serum muscle proteins over 3 days. The study was arranged in two separate cohorts of placebo vs $5 \mathrm{mg}$ daprodustat, and placebo vs $50 \mathrm{mg}$ daprodustat (Additional file 5: Table S2 describes demographic data). There were 15 total adverse effects; none serious or thought to be compound-related, with the most frequent being exercise-induced arm muscle pain (myalgia, Additional file 6: Table S3). Both MVC force and range of motion were reduced by exercise but were not altered with either dose of daprodustat (Fig. 4b, Additional file 4: Figure S3b). However, increases in serum creatine kinase activity after exercise were significantly lower in individuals administered $50 \mathrm{mg}$ daprodustat (Fig. 4c).

We pursued an aptamer-based proteomics approach to identify additional circulating markers of muscle injury and the effects of daprodustat treatment in serum from the placebo and $50 \mathrm{mg}$ daprodustat groups (Somalogic, Inc.). Comparison of pre- and post-injury serum in the placebo group revealed that 9 of 11 proteins increased with exercise were associated with skeletal muscle and were all lowered by daprodustat (Fig. 4d, Additional file 7: Table S4). These changes are consistent with the mouse data, suggesting a reduction in tissue damage after muscle injury with HIF prolyl hydroxylase inhibition.

\section{Discussion}

PHD regulation of HIF1 $\alpha$ has been implicated in the protection of the heart, brain, and liver after an ischemic insult $[14,29,30]$. The mechanism of this protection appears to vary by tissue type and often reflects the effect of chronic HIF1 $\alpha$ activation, robust target gene activation, and tissue remodeling after PHD protein knockout. Compared to these reports, we were surprised by both the acute nature of the skeletal muscle protective effect and its modest transcriptional response in muscle in our preclinical system. Higher doses $(30 \mathrm{mg} / \mathrm{kg}$ ) of GSK360 have been associated with HIF1 $\alpha$ target gene activation in muscle [14], but our studies identified a more sensitive myeloid population that drives muscle protection in an iNOS-dependent manner without large scale transcriptional responses. iNOS is required for the efficient recovery of skeletal muscle after injury in mice [23], but to our knowledge, this is the first time that iNOS has been implicated in an acute protective role after muscle injury. In skeletal muscle ischemia-reperfusion injury, iNOS is generally associated with a delayed inflammatory response and leads to high NO levels, membrane lysis, and skeletal muscle breakdown [31]. A complex balance exists between protective and detrimental effects of $\mathrm{NO}$ dependent upon the time and extent of $\mathrm{NO}$ release [32]. Therefore, we presume that the modest inflammatory response and NO generation associated with eccentric contraction-induced muscle injury leads to a greater protective role for $\mathrm{NO}$ generated through iNOS. We demonstrate that the macrophage HIF1 $\alpha$ compartment is essential for the protective effect of PHD inhibition, which is consistent with their presumed role in membrane repair after eccentric contraction-induced muscle injury [20]. The exact mechanism by which the hypoxic response generated by PHD inhibition in macrophages is driving the protection and recovery from the muscle injury is as yet undetermined, but it is likely to either be remodeling the inflammatory response or accelerating satellite cell activation [33]. GSK360 and daprodustat are both non-selective inhibitors of PHD1, 2 , and $3[14,34]$, and polarized macrophages express all three PHD isoforms [35], making determination of which isoform(s) are required for muscle protection difficult without more detailed studies. In preliminary experiments, GSK360 treatment of M1/M2 polarized 


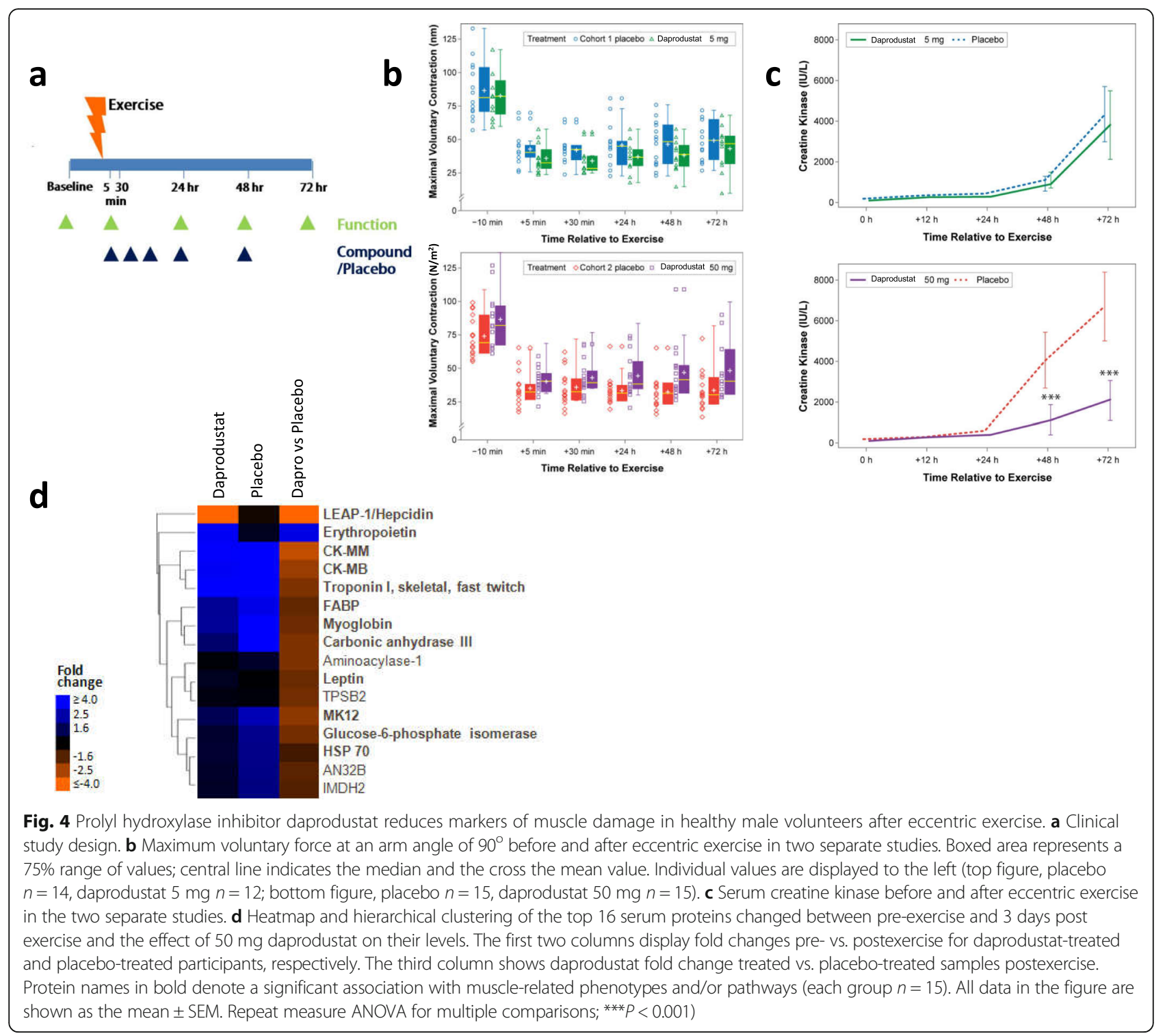

BMDM cells resulted in the upregulation of both inducible PHDs (PHD2 and PHD3) in M1 but not M2 macrophages (data not shown). This would suggest reflex compensation from elevated HIF1 $\alpha$ in M1 cells but does not suggest which key $\mathrm{PHD}(\mathrm{s})$ are driving this response.

While healthy volunteer data did not recapitulate the functional protection of PHI seen in mice, circulating markers of muscle damage were reduced by daprodustat in the highest dose group. This is possibly due to differences between these two systems. The cause of voluntary strength deficits in people after eccentric exercise are not well understood [36] and are associated with modest changes in histological and inflammatory markers of muscle damage [37, 38] compared to the more robust damage we observed in mouse models. Force deficits in people can also occur without elevation of circulating markers of muscle damage after preconditioning exercise
[39], suggesting that mechanisms independent of histological damage, such as excitation-contraction coupling failure [36], may be the determinants of human functional deficits. Further, voluntary muscle contraction in humans removes the contribution of factors such as muscle pain to reductions in arm strength, which are not captured by involuntary electrical stimulation in the mouse. We also note that the volunteers used in this study were somewhat overweight (mean BMI 26-28.5), possibly due to the entry requirement for no regular exercise, a factor that could also influence the outcome of the study.

Notably, the circulating signature of muscle-related proteins elevated by exercise and decreased by daprodustat treatment in human volunteers is remarkably similar to that observed in muscular dystrophy patients [40]. This likely reflects similarities between muscle injury after eccentric exercise in healthy individuals and muscle 
injury due to structural fragility in dystrophic patients. Further research is underway to understand if muscle protection through PHD inhibition may provide protection in the background of muscular dystrophy.

\section{Conclusion}

We have uncovered a novel connection between HIF1 $\alpha$ and iNOS that contributes to muscle protection after exercise-driven injury in mice. Evidence suggests that some elements of this protective effect may also be competent in healthy volunteers after eccentric injury, although there are clear differences in the magnitude of response between mouse and man. Our data implicates the HIF signaling axis as an important modulator of inflammatory activity during skeletal muscle repair and injury and further indicates that this mechanism may be of therapeutic importance in the treatment of acute muscle injury.

\section{Materials and methods Preclinical methods Animals}

Myeloid cell-specific Hif1a null (HIF1a KO) (B6.129Hif1atm3Rsjo/J x B6.129P2-Lyz2tm1(cre)Ifo/J), iNOS KO (B6.129P2-iNOStmLau/J), and normal C57Bl6/NJ mice were purchased from Jackson labs. Male adult mice (3-4 months-old) were housed individually (12 h:12 h day:night cycle) in a temperature- and humidity-controlled environment $\left(73.5 \pm 5.5^{\circ} \mathrm{F}\right.$ and $50 \pm 20 \%$, respectively) and fed standard chow (LabDiet 5001, Purina Mills, LLC, St. Louis, MO) and water ad libitum. Mice were utilized for experimentation following a 7-day acclimation period and randomized into treatment groups by body weight. All studies were conducted in accordance with the GSK Policy on the Care, Welfare and Treatment of Laboratory Animals. All protocols were reviewed and approved by the Institutional Animal Care and Use Committee of GSK.

\section{In vivo reagents}

GSK1120360 (GSK360) was formulated in 0.5\% hydroxypropylmethylcellulose (Acros Organics, Lot\#A0320015, Code 244020010, CAS 9004-65-3) and 0.1\% Tween80 (Fisher, Lot\#141362, Cat\# T164-500) (HPMC:Tween) vehicle and administered (PO) daily at specified doses beginning on the day of eccentric damage (with the exception of the experiment in Fig. 1b, with timings as indicated). The iNOS-specific inhibitor $1400 \mathrm{~W}(20 \mathrm{mg} / \mathrm{kg})$ and recombinant mouse erythropoietin (EPO) $(20 \mu \mathrm{g} / \mathrm{kg})$ were injected subcutaneously in saline-based vehicles. $1400 \mathrm{~W}$ was administered 2-h prior to eccentric damage, while EPO was dosed daily for 3 days leading up to the day of damage. For EPO neutralization, $600 \mu \mathrm{g}$ of a monoclonal antibody targeting EPO (R\&D Systems, MAB959) was injected SC at days -3 and 1 prior to eccentric damage. For EPO depletion studies, $200 \mu \mathrm{g} / \mathrm{kg}$ of a soluble EPO receptor (R\&D Systems, 1390-ER-050) was injected SC twice a day for 3 days prior to eccentric damage.

\section{In vivo muscle contractility}

Mice were placed on a warming plate $\left(30-32{ }^{\circ} \mathrm{C}\right)$ and anesthetized using $2 \% / \mathrm{L} \mathrm{O}_{2}$ isoflurane. Right hind limbs were first shaved and then pinned at the knee at $90^{\circ}$ with foot strapped into a mouse "shoe" which doubles as a motor arm and force transducer (Aurora Scientific Instruments 305C, Aurora). Platinum sub-dermal electrodes (Grass Instruments; West Warwick, RI) were inserted dorsally and ventrally to the femur for field stimulation of the sciatic nerve. Because the mouse gastrocnemius, plantaris, and soleus muscles are larger and stronger as a complex than the ankle dorsiflexors (TA and EDL), hind limbs produced a net plantarflexion response to sciatic activation. Tetanic stimuli of $150 \mathrm{~Hz}$ at $200 \mu$ s pulse width (with $2 \mathrm{~mA}$ current and $15 \mathrm{~V}$ ) for $0.8 \mathrm{~s}$ were recorded as maximal isometric limb forces while single $200 \mu$ s pulses were used to elicit maximal twitch force values. Muscle contractility was evaluated immediately prior to injury (baseline force day 0 ), day 1 post-injury, and then at varying longitudinal points thereafter (as indicated).

\section{Eccentric contraction-induced muscle injury}

To induce muscle injury, mice were positioned as described above and initial baseline maximal twitch and tetanic limb force measurement were performed. After a 2-min rest interval, mice underwent a unilateral eccentric muscle contraction protocol in which the hind limb plantarflexor muscles were forcibly lengthened using the automated footplate $\left(30^{\circ}\right.$ angular rotation, $\left.1800^{\circ} / \mathrm{s}\right)$ while being concomitantly stimulated to contract $(150 \mathrm{~Hz}$; $200 \mu$ s pulse width; $2 \mathrm{~mA}$ and $15 \mathrm{~V}$ ). A bout of eccentric exercise consisted of 1 forced lengthening contraction every $10 \mathrm{~s}$ for a total of 60 repetitions.

\section{Histology}

With the muscle still in situ, an ink mark was made on the muscle belly to serve as a guide for reproducible collection of sections for analysis. Following excision, the gastrocnemius muscles were oriented to provide cross sectional fiber profiles when sectioned. A small drop of OCT media (Sakura Finetek, USA) was placed on a cork circle and the muscle was stabilized by placing the distal end of the muscle into the media. The muscle was maintained in a vertical position while immersing it into liquid nitrogen cooled isopentane for 10 to $15 \mathrm{~s}$ until frozen; samples were held at $-80{ }^{\circ} \mathrm{C}$ until sectioned. Ten-micrometer frozen sections were prepared in a cryostat and mounted on glass slides. Tissue sections 
were fixed with $4 \%$ paraformaldehyde diluted in PBS for $5 \mathrm{~min}$ at room temperature immediately prior to immunohistochemistry (IHC). Immunostaining was carried out on an automated system (Ventana Medical, USA). Briefly, the sections were blocked with 10\% normal goat serum for $32 \mathrm{~min}$ at $37^{\circ} \mathrm{C}$ prior to application of a cocktail of anti-laminin and anti-albumin antibodies. Anti-laminin (Ab44941, Abcam, USA) and anti-albumin antibody (Ab19196, Abcam, USA) were applied at $5 \mu \mathrm{g} /$ $\mathrm{ml}$ and 1:10000 respectively for $1 \mathrm{~h}$ at $37^{\circ} \mathrm{C}$. Albumin and laminin were detected using species-specific fluorophore conjugated secondary antibodies. Albumin immunoreactivity was demonstrated using an Alexa594 conjugate (A-11037, Invitrogen, USA) at $5 \mu \mathrm{g} / \mathrm{ml}$ and laminin expression using an Alexa488 conjugate (A-11006, Invitrogen, USA) at $10 \mu \mathrm{g} / \mathrm{ml}$; incubation in detection antibodies was for $45 \mathrm{~min}$ at $37^{\circ} \mathrm{C}$. Following automated wash steps the nuclei were stained using DAPI (D-3571, Invitrogen, USA) then cover slipped using an aqueous mounting media (P36961, Invitrogen, USA). Stained sections were imaged at $\times 20$ magnification on the Zeiss AxioScan.

\section{Quantitative image analysis}

A bespoke image analysis algorithim was developed (Definiens, Inc., USA) for use with Definiens Architect XD (Definiens AG, Germany) software. The entire cross-sectional area of the scanned gastronemius muscle was included in the analysis using the above software. Laminin expression was used to isolate individual cross-sectional myofibers. These individual fibers were assessed for their expression of albumin (Optical Density) as well as additional morphometric parameters (e.g., perimeter, area, roundness, myonuclei count and intracellular distribution). Individual fiber data were exported to CSV files for statistical analysis.

\section{Gene expression analyses}

Mouse gastrocnemius samples were collected and flash frozen in liquid nitrogen then stored at $-80{ }^{\circ} \mathrm{C}$. For RNA isolation, $\sim 100 \mathrm{mg}$ samples were used in $1 \mathrm{ml}$ Trizol and isolation was done according to the manufacturers protocol (Ambion Cat \#15596-068). Purified RNA went through Qiagen RNA clean up kit (Cat \#74204) and eluted in $50 \mu \mathrm{l}$ elution buffer. For each sample $2 \mu \mathrm{g}$ total RNA was submitted to Q2 Solutions for Affymetrix analysis, using Affymetrix Gene Chip Mouse Genome 430A 2.0 Array. Raw data files (CEL files) were then pre-processed using the RMA (Robust Multi-chip Average) pipeline [41] in combination with the most current re-annotated probeset definitions [42]. To determine differential mRNA expression, a linear model was fit [43, 44]. The false discovery rate (FDR) was computed as an adjusted $P$ value [45] to account for multiple testing and a cut-off of $10 \%$ FDR was used to define differential expression. For BMDM work, RNA was isolated from BMDM using a Turbo Capture plate and kit (Qiagen) per manufacturer's protocol. iNOS expression was quantitated by Taqman qRT-PCR using. Housekeeping genes, Ppia (PRIMERS) and Rpl-p0 (PRIMERS), were used to normalize expression. Data are presented as $\Delta \mathrm{Ct}$.

\section{Plasma assessment of mouse erythropoietin}

Plasma EPO and VEGF concentrations were measured using mouse/rat EPO/VEGF serum/plasma multiplexed immunoassay (Meso Scale Discovery, Gaithersburg, $\mathrm{MD}$ ) according to manufacturers' protocols.

\section{Bone marrow-derived macrophage generation}

Tibia and femurs from wild type mice were stripped of muscle tissue and the marrow flushed using a 24-G needle and syringe filled with basal culture media (RPMI 1640 + Glutamax (Gibco), 10\% fetal bovine serum (Gibco), 5\% horse serum (Gibco), and 1\% antibiotic/antimycotic (Gibco)). Isolated marrow cells were gently triturated to a uniform suspension and then filtered through a $40-\mu \mathrm{m}$ cell strainer. The cell suspension was then centrifuged ( $500 \mathrm{~g}$ for $5 \mathrm{~min}$ ) and the resultant pellet resuspended in $1 \times$ Red Blood Cell Lysis Buffer (Miltenyi Biotec) and gently agitated for 3 min to lyse red blood cells. The cell suspension was centrifuged, and the resultant pellet resuspended in basal culture media to a final cell concentration of $10^{7}$ cells $/ \mathrm{ml}$. Cells were cultured at an initial density of $1.5 \times 10^{6}$ in $10 \mathrm{~cm}$ bacteriological dishes in basal culture media supplemented with $10 \mathrm{ng} / \mathrm{ml}$ macrophage colony stimulating factor (MCSF; R\&D Systems). At day 4 post-plating, cells were supplemented with basal culture media $+10 \mathrm{ng} / \mathrm{ml}$ MCSF. BMDMs were polarized and treated with GSK360 on day 7. To generate M1 macrophages, basal culture media was supplemented with $20 \mathrm{ng} / \mathrm{ml}$ recombinant mouse IFN $\gamma$ (Millipore, cat \#407320) $+100 \mathrm{ng} / \mathrm{ml}$ of lipopolysaccahride (LPS; SigmaAldrich, cat\# L5293). For M2 activation, basal culture media was supplemented with $20 \mathrm{ng} / \mathrm{ml}$ recombinant mouse IL-4 (R\&D Systems, cat\# 404-ML). For naïve, M0 macrophages, only basal culture media was used. GSK360 was then added to designated cultures at a final concentration of $10 \mu \mathrm{M}$, with DMSO added as a vehicle control. BMDM were cultured for a final $24 \mathrm{~h}$ at $37{ }^{\circ} \mathrm{C}$ then isolated by gently pipetting using ice cold PBS (Gibco, pH 7.4) following incubation on ice for $10 \mathrm{~min}$. The suspension was then centrifuged, and the resultant cell pellets processed for either RT-PCR or Western blotting.

\section{Adoptive transfer of BMDM}

Bone marrow from the tibia and femurs of myeloid HIF1 $\alpha \mathrm{KO}$ and HIF1 $\alpha$ WT littermates were used to culture bone marrow-derived macrophages (BMDMs) as 
described above. Seven days following initial culture, BMDMs were lifted from culture dishes with Accutase (Gibco). Cell suspensions were pooled in $50-\mathrm{ml}$ conical tubes, centrifuged (500g for $5 \mathrm{~min}$ ), resuspended in serum-free RPMI 1640, counted for cell density numbers, and centrifuged again to pellet. BMDMs were then labeled with PKH67 Green according to manufacturer's protocol (Sigma-Aldrich). Once labeled, BMDMs were rinsed three times with sterile saline and resuspended at two million cells per $100 \mu \mathrm{l}$.

Myeloid HIF1 $\alpha$ KO recipient animals were randomized by body weight and initial tetanic force production. Animals were anesthetized, and the eccentric exercise protocol was performed as described above. Immediately following the exercise protocol, $100 \mu \mathrm{l}$ of BMDM suspension (either HIF $1 \alpha$ KO or HIF1 $\alpha$ WT) were introduced directly to the injured gastrocnemius via intramuscular injection. Animals were then treated with either vehicle or GSK360 ( $N=8$ for each group). At $24 \mathrm{~h}$ post-eccentric damage, force production was assessed as described above.

\section{FACS analysis}

Muscle tissues were minced and treated with LiberaseTM (Roche) and Dnase I for $2 \mathrm{~h}$ at $37{ }^{\circ} \mathrm{C}$. After digestion, tissue homogenates were filtered through $70 \mu \mathrm{m}$ strainers for cell suspension. For flow cytometry assay, cells were first treated with Fc Block (1:1000; BioLegend), followed by staining surface antigens with UV viability dye (Zombie 1:400; BioLegend), CD11b-PE (M1/ 70, 1:400), Ly6C-PerCP/Cy5.5 (HK1.4, 1:400), CD45-PE/ Cy7 (30-F11, 1:1000) and I-A/I-E (M5/114.15.2, 1:400). Macrophages were labeled with F4/80 mAb (45-4801-80, ThermoFisher). Data were acquired on FACS Aria II and analyzed by FlowJo V10.2.

\section{Western blotting}

BMDM cell pellets were resuspended in T-PER lysis buffer (Life Technologies) supplemented with Halt ${ }^{\text {tw }}$ protease inhibitor cocktail and EDTA (Life Technologies). Suspensions were incubated on ice for 20 min with periodic vortexing, then centrifuged to clarify. Supernatants were assayed for protein content using a microplate BCA assay (ThermoFisher) per manufacturer's protocol. For each sample, a total of $10 \mu \mathrm{g}$ of protein were separated on a $4-12 \%$ Bis-Tris Bolt ${ }^{\text {ti }}$ SDS-PAGE gels (Life Technologies) and transferred to nitrocellulose membranes using an iBlot2 (Life Technologies).

Gastrocnemius muscles were dissected and snap frozen. Approximately $50 \mathrm{mg}$ of frozen tissue was lysed in T-PER lysis buffer (Life Technologies) supplemented with Halt ${ }^{\mathrm{Tm}}$ protease inhibitor cocktail and EDTA (Life Technologies) using an MPBio Bead Blender. Lysates were incubated on ice for $20 \mathrm{~min}$ and then centrifuged to clarify. Supernatants were assayed for protein content using a microplate BCA assay (ThermoFisher) per manufacturer's protocol. For each sample, $50 \mu \mathrm{g}$ of protein was separated on $4-12 \%$ Bis-Tris Bolt ${ }^{\mathrm{tw}}$ SDS-PAGE gels (Life Technologies) and transferred to nitrocellulose membranes using an iBlot2 (Life Technologies). The same sham samples were run on each blot for both $1 \mathrm{~h}$ and $24 \mathrm{~h}$ time points for comparison to baseline.

Membranes were blocked using Superblock TBS + Tween-20 (ThermoFisher) for $1 \mathrm{~h}$ at room temperature. Primary antibodies, diluted in Superblock TBS + Tween-20, were added to the membranes and incubated overnight at $4{ }^{\circ} \mathrm{C}$ on a platform rocker. Membranes were washed three times for $15 \mathrm{~min}$ at room temperature with TBS $+0.5 \%$ Tween-20 (ThermoFisher). Secondary antibodies, diluted in Superblock TBS + Tween-20, were then added to the membranes and incubated for $4 \mathrm{~h}$ at room temperature, protected from light. Membranes were then washed as before and imaged on an Odyssey CLx (Li-Cor Systems). Mouse anti-iNOS (BD Transduction Labs, clone 6/iNOS) used at a dilution of 1:1000; rabbit anti-Rab5 (Cell Signaling Technologies) used at a dilution of 1:1000; goat anti-HIF1a (R\&D Systems) used at a dilution of 1:1000; donkey anti-mouse 680RD secondary antibody (Li-Cor Biosystems) used at a dilution of 1:10,000; donkey anti-rabbit 800CW (Li-Cor Biosystems) used at a dilution of 1:10,000; donkey anti-goat $800 \mathrm{CW}$ (Li-Cor Biosystems) used at a dilution of $1: 10,000$.

\section{HIF1a ELISA}

BMDMs polarized to M0, M1, or M2 (as described above) were treated with GSK360 for $24 \mathrm{~h}$ ( $n=2$ per dose point). Cells were then lysed using T-PER lysis buffer (Life Technologies) supplemented with Halt ${ }^{\mathrm{tw}}$ protease inhibitor cocktail and EDTA (Life Technologies). Resultant suspensions were incubated on ice for $20 \mathrm{~min}$ with periodic vortexing then centrifuged to clarify. Supernatants were assayed for protein content using a microplate BCA assay (ThermoFisher) per manufacturer's protocol. Samples were run in triplicate in a DuoSet HIF1 $\alpha$ ELISA (R\&D Systems), following the standard protocol. Data was captured with a SpectraMax spectrophotometer and analyzed using GraphPad Prism 7.0.

\section{Preclinical statistical analysis}

For each experiment, the means and SEM of the parameters measured were determined. Statistical analyses were performed using Student's $t$ test in single-factorial designs. For multifactorial study designs, one- or two-way ANOVA was used as appropriate. Tukey's (for one-way ANOVA) or Bonferroni (for two-way ANOVA) post hoc test was applied when significant differences were found. Testing for normal variance was performed 
and confirmed. For mouse experiments, the number of mice per group required to detect biologically significant effect sizes was calculated using an appropriate statistical sample-size formula and indicated in the biometrical planning section of the animal license submitted to the governing authority. Blinding was not done during animal group allocation but was done for some measurements made in the study (i.e., histology). No specific exclusion criteria were applied, as inbred strains, which display uniform phenotypic characteristics, were used exclusively. Analyses were carried out with SigmaPlot v.12 software (Systat Software $\mathrm{GmbH}$, Erkrath) or GraphPad Prism software (GraphPad Software, San Diego). $P<0.05$ was considered statistically significant.

\section{Clinical methods}

\section{Eccentric exercise-induced muscle damage in healthy} volunteers

This was a repeat dose, two-cohort, randomized, placebo-controlled, parallel group study in healthy volunteers (Clinicaltrials.gov reference NCT02231190). In each cohort, approximately 30 subjects were enrolled and randomized into a 1:1 ratio between daprodustat and placebo groups (study CONSORT diagram, Additional file 4: Figure S3). The study protocol, any amendments, the informed consent, and other information that required pre-approval were reviewed and approved by an investigational center institutional review board, in accordance with the International Conference on Harmonization of Technical Requirements for Registration of Pharmaceuticals for Human Use (ICH) Good Clinical Practice (GCP) and applicable country-specific requirements, including United States (US) 21 Code of Federal Regulations (CFR) 312.3(b) for constitution of independent ethics committees.

This study was conducted in accordance with $\mathrm{ICH}$ GCP and all applicable subject privacy requirements and the ethical principles that are outlined in the Declaration of Helsinki 2008. The study was monitored in accordance with ICH E6, Section 5.18. Investigators were trained to conduct the study in accordance with GCPs and the study protocol as defined in ICH E3, Section 9.6. Written commitments were obtained from investigators to comply with GCP and to conduct the study in accordance with the protocol.

Written informed consent was obtained from each subject prior to the performance of any study-specific procedures. The investigator agreed to provide the subjects as much time as necessary to review the document, to inquire about details of the trial, and to decide whether or not to participate in the study. The informed consent was signed and dated by the study subjects and by the person who conducted the informed consent discussion. Electronic case report forms were provided for each subject's data to be recorded.

\section{Study methods}

Subjects who met all screening criteria were enrolled and admitted to the clinical test center 1 day before the exercise protocol. Subjects who had a creatine kinase (CK) within the normal range at baseline participated in the study. Ten minutes before the exercise protocol, range of motion, muscle pain/soreness, and MVC were measured. The exercise protocol was then conducted on the same arm (details below). Subjects who achieved a deficit from baseline of at least $40 \%$ in their MVC were randomized to either daprodustat or placebo and entered the post-exercise phase. Fifteen minutes after completion of eccentric exercise, each subject started to receive a total of five oral doses of daprodustat or placebo on day 1 ( $0 \mathrm{~h}, 4 \mathrm{~h}, 8 \mathrm{~h})$, day $2(24 \mathrm{~h})$, and day 3 (48 h). Functional assessments performed pre-exercise were repeated at $5 \mathrm{~min}, 30 \mathrm{~min}, 24 \mathrm{~h}, 48 \mathrm{~h}$, and $72 \mathrm{~h}$ post-exercise. Subjects were discharged from the unit on day 4 and returned for a follow-up visit 7-10 days later.

The study was performed in two separate cohorts. In cohort 1 , subjects were randomized to either placebo or $5 \mathrm{mg}$ daprodustat. In cohort 2, subjects were randomized to either placebo or $50 \mathrm{mg}$ daprodustat. The progression to the second cohort, termination of study, or a repeat of the same dose level was based on the feasibility of the eccentric exercise protocol and the totality of the data. The data sets reviewed, at minimum, consisted of safety data and observed MVC at $90^{\circ}$ of flexion. This study was double-blinded with respect to the subjects, the investigator and site staff (with the exception of the site pharmacist). GlaxoSmithKline (GSK) was un-blinded throughout the study. This study was performed in compliance with Good Clinical Practices and GlaxoSmithKline Standard Operating Procedures for all processes involved, including the archiving of essential documents. Anonymized individual participant data and study documents can be requested for further research from www.clinicalstudydatarequest.com.

\section{Inclusion criteria}

Subjects were eligible for inclusion in this study only if all of the following criteria were met:

1. Males between 18 and 35 years of age inclusive, at the time of signing the informed consent.

2. Healthy as determined by a responsible and experienced physician, based on a medical evaluation including medical history, physical examination, laboratory tests, and cardiac monitoring. 
3. Body weight $\geq 70 \mathrm{~kg}$ and $\mathrm{BMI}$ within the range 22.0 to 34.0 (inclusive).

4. Capable of giving written informed consent, which included compliance with the requirements and restrictions listed in the consent form.

5. ALT, alkaline phosphatase, and bilirubin $\leq 1.5 \times$ ULN .

6. QTc $<450 \mathrm{~ms}$ based on single or average QTc value of triplicate values obtained over a brief recording period.

7. At least a 1 year history of no regular (2-3 times per week) exercise and no heavy exertion within past week.

8. No strenuous exercise involving the arms during the last 10 weeks.

\section{Eccentric exercise protocol}

Each subject performed maximal eccentric contractions of the elbow flexors of one arm against a lever arm moving at a constant (isokinetic) angular velocity over a limited range of motion. The aim of this procedure was to elicit at least a $40 \%$ decline in isometric strength from the start to the end of the exercise. To take account of variability in performance between individuals, the exercise procedure was repeated up to three times in total to elicit this deficit. Subjects not manifesting at least a $40 \%$ decline in isometric strength were excluded from the study. Each subject will be placed on a Cybex with the lever arm fixed so that the elbow was at $90^{\circ}$ flexion. The subject was then asked to provide an MVC of the elbow flexors by pulling against the lever arm while force production is measured. The MVC was measured three times in succession and the highest value recorded. This value was used as a reference assessment of volunteer performance in the subsequent exercise protocol. After a 2-min rest, subjects were asked to perform five sets of six maximal eccentric contractions of the elbow flexors of the non-dominant arm to induce local muscle inflammation, pain and transient functional deficits. Each eccentric contraction started at an elbow angle of $90^{\circ}$ flexion and volunteers were asked to pull against the lever arm before the lever arm extended to $180^{\circ}$ at a constant angular velocity of $60^{\circ} / \mathrm{s}$. Subjects were verbally encouraged to maximally resist the lowering lever arm of the ergometer during the eccentric movement flexion. At the end of each eccentric contraction, each subject was instructed to relax and the lever arm returns to the $90^{\circ}$ flexion position at an angular velocity of $10^{\circ} / \mathrm{s}$ before the next eccentric contraction. Each bout of 6 contractions was separated by 2 min of resting recovery. At the end of the five sets, after a 2-min rest period, the subject was asked to provide another MVC. At this point, the instructor calculated the acute post-exercise MVC deficit. If post-exercise MVC was $<40 \%$ lower than pre-exercise, this triggered a second round of 30 eccentric contractions.
If post-exercise MVC was $>40 \%$ lower than pre-exercise, the exercise was considered complete. For the subjects entering a second round of eccentric exercise, they repeated five sets of six maximal eccentric contractions with a 2 -min rest period between sets as above. At the end of the five sets, after a 2-min rest period, the subjects were asked to provide an MVC. If post-exercise MVC was $>40 \%$ lower than pre-exercise, the exercise was considered complete. There was provision to go to a third round of exercise if post-exercise was $<40 \%$ of pre-exercise MVC but every subject in the study achieved a $>40 \%$ deficit within two exercise rounds.

\section{Study objectives and endpoints}

The primary objective of the study was to evaluate the protective effects of daprodustat on eccentric exercise-induced muscle injury by measuring MVC of the exercised arm up to $72 \mathrm{~h}$ after completion of eccentric exercise in subjects treated with daprodustat in comparison to subjects treated with placebo. Secondary objectives included assessment of the safety and tolerability of daprodustat by measuring adverse events, vital signs (systolic and diastolic blood pressure, pulse rate, and respiratory rate), 12-lead electrocardiogram (ECGs) (heart rate, PR, QRS, QT, and QTcF), and laboratory parameters (Additional file 6: Table S3). Another secondary objective was to evaluate the protective effects of daprodustat on deficits in arm range of motion after eccentric exercise by measuring change in degree of motion and resting arm angle from post-exercise up to $72 \mathrm{~h}$ after completion of eccentric exercise in subjects treated with daprodustat in comparison to subjects treated with placebo.

\section{Measurement of MVC isometric strength}

Strength was assessed in the non-dominant arm by a maximal isometric (fixed length) contraction. The subject was placed on a Cybex ergometer (Medway, MA) in a semi-supine position grasped the dynamometer handle with the wrist in a supinated position. MVC was measured at both $90^{\circ}$ and $150^{\circ}$ of extension at the elbow $\left(90^{\circ}\right.$ and $30^{\circ}$ of flexion respectively). While the subject was seated, the lever arm was fixed so that the elbow was at the appropriate angle $\left(90^{\circ}\right.$ and $150^{\circ}$ of extension $/ 90^{\circ}$ and $30^{\circ}$ of flexion respectively). The subject was then asked to provide a maximal voluntary contraction of the elbow flexors by pulling against the lever arm while force-production was measured. The MVC was measured three times in succession at each angle, with a $30 \mathrm{~s}$ rest between attempts. The highest value at each angle was recorded.

\section{Measurement of elbow range of motion (ROM)}

A manual goniometer was used to measure the range of motion at the elbow. Joint angles were measured at rest, at maximum extension and at maximum flexion. Measurements were taken twice for each joint angle and the 
mean value of the two measurements was used to calculate the ROM by subtracting the flexion angle from the extension angle.

\section{Somascan serum analysis}

Baseline and $72 \mathrm{~h}$ serum samples from Cohort 2 were examined with an aptamer-based proteome analysis screen (SOMAlogic Inc., SOMAscan ${ }^{\circ}$ Boulder, CO, Fig. 3d and Additional file 7: Table S4). Protein concentration data was $\log 2$ transformed and normalized using quantile normalization [44]. To determine differential expression, a linear model was fit [43] taking into account the treatment effect as well as the pair wise design (i.e., samples from the same individual before and after treatment). The false discovery rate (FDR) was computed as an adjusted $P$ value to account for multiple testing and a cutoff of $10 \%$ FDR as well as an absolute fold change cutoff of 1.5 or greater was used to define differential expression. All analyses were done using the R/ Bioconductor and ggplot2 software packages [46].

\section{Clinical statistical analysis}

Clinical primary endpoints were analyzed using a ANCOVA model with a fixed term of treatment regimen, visit, interaction of regimen and visit, baseline endpoint as a covariate and subject as a random effect. Point estimate and its $95 \%$ confidence interval were obtained from the model for comparisons of interests (e.g., active doses versus placebo at each visiting time point). Biomarkers, including serum CK activity, after logarithm transformation, were analyzed with a repeat measure ANOVA with fixed terms of regimen, visit and interaction of regimen and visit, subject as random effects.

\section{Additional files}

Additional file 1: Figure S1. Prolyl hydroxylase inhibitors protect muscle at doses that raise EPO but not VEGF. (a) Effect of different daily doses of GSK360 on contraction induced injury in mice. Mice were treated with vehicle or the indicated dose of GSK360 daily. Data are normalized to pre-damage force $(n=8)$. (b) Effect of co-treatment with EPO neutralizing antibody and GSK360 for 3 days on circulating red blood cells and hematocrit $(n=8)$. All data in the figure are shown as the mean \pm SEM. Two-way ANOVA followed by Holm-Sidak's test corrected for multiple comparisons; ${ }^{* *} P<0.01$ as compared to vehicle dosed cohort. (PDF $224 \mathrm{~kb}$ )

Additional file 2: Figure S2. Prolyl hydroxylase inhibitors do not alter transcriptional profiles in skeletal muscle. (a) Transcriptional profile in mouse gastrocnemius muscle $9 \mathrm{~h}$ after injury with $10 \mathrm{mg} / \mathrm{kg}$ GSK360 or vehicle. After correcting for multiple testing, no statistically significant changes were detected. (b) Relative tissue concentration of GSK360 after $4 \mathrm{~h}$ of IV infusion $(n=3)$. (c) Flow cytometry quantification of F4/80 positive macrophages in muscle 0-14 days after muscle injury with $10 \mathrm{mg} / \mathrm{kg}$ GSK360 or vehicle $(n=4)$. (d) Quantification of HIF1a in polarized murine bone marrow-derived macrophages (BMDM) following $24 \mathrm{~h}$ of GSK360 treatment. Protein extracts from BMDMs were generated $(n=2)$. HIFla was quantified via DuoSet ELISA (R\&D Systems) with protein extracts. All data in the figure are shown as the mean \pm SEM. Two-way
ANOVA followed by Holm-Sidak's test corrected for multiple comparisons: ${ }^{*} P<0.01$ as compared to vehicle dosed cohort. (PDF $257 \mathrm{~kb}$ )

Additional file 3: Table S1. Excel file of Affymetrix Gene Chip mRNA levels from mouse gastrocnemius muscle 3,6 and 9 hrs after injury with $10 \mathrm{mg} / \mathrm{kg}$ GSK360 vehicle. Table shows comparison of expression levels between vehicle and GSK360 treated muscle. (XLSX $1910 \mathrm{~kb}$ )

Additional file 4: Figure S3. Daprodustat does not alter arm range of motion after eccentric exercise. (a) Effect of different daily doses of daprodustat on contraction induced injury in mice. Mice were treated with vehicle or the indicated dose of daprodustat daily. Data are normalized to pre-damage force $(n=9)$. (b) Arm range of motion, measured with a manual goniometer was recorded 10 min pre-exercise and $5 \mathrm{~min}, 30 \mathrm{~min}, 24 \mathrm{~h}, 48 \mathrm{~h}$, and $72 \mathrm{~h}$ post exercise. The boxed area represents a $75 \%$ range of values with the central line indicating the median and the cross the mean value. Individual values are displayed to the left (top panel placebo vs GSK863 $5 \mathrm{mg}(n=14, n=12)$, bottom panel placebo vs GSK863 $50 \mathrm{mg}$ (both $n=15)$ ). (PDF $270 \mathrm{~kb}$ )

Additional file 5: Table S2. Demographic data for the two healthy volunteer studies. Twenty-six healthy volunteers were randomized in Cohort 1, 30 were randomized in cohort 2, with approximately 1:1 ratio of daprodustat vs. placebo. All randomized subjects completed the study, and the data from all randomized subjects were included in the study analyses. Overall, demographic characteristics were similar among the placebo and GSK127863 groups. No violation of the treatment assignment or broken blinding occurred in this study. No significant protocol deviations were found during the study. (PDF $200 \mathrm{~kb}$ )

Additional file 6: Table S3. Summary of adverse events. No serious adverse events (SAEs) were reported in this study. Fifteen subjects (26.8\%) reported adverse events (AEs). The most commonly reported AEs ( $>1$ subject) were myalgia in nine subjects $(16.1 \%)$, headache in two subjects (3.6\%), and peripheral swelling in two subjects (3.6\%). Myalgia was largely limited to arm pain during and after the exercise. Single events of vomiting and pollakiuria, both in the placebo group, were not considered by the investigator as related to study drug. No AEs led to withdrawal of subjects from this study. There were no clinically significant findings of vital signs, ECG or clinical laboratory parameters. (PDF $196 \mathrm{~kb}$ )

Additional file 7: Table S4. Effect of daprodustat on serum protein changes pre- and 3 days post exercise. Excel file for SOMAscan ${ }^{\circledR}$ (SOMAlogic Inc.) aptamer-based proteome analysis of serum comparing baseline (pre-injury) and $72 \mathrm{~h}$ post-injury serum samples from Cohort 2 of the healthy volunteer study. Protein concentration data was log2 transformed and normalized using quantile normalization [44]. To determine differential expression, a linear model was fit [43] taking into account the treatment effect as well as the pair wise design (i.e., samples from the same individual before and after treatment). (PDF $1428 \mathrm{~kb}$ )

\section{Abbreviations}

BMDM: Bone marrow-derived macrophage; EPO: Erythropoietin; HIF: Hypoxiainduced factor; MVC: Maximum voluntary contraction; NOS: Nitric oxide synthase; PHD: Prolyl hydroxylase; PHI: Prolyl hydroxylase inhibitor

\section{Acknowledgements}

The authors would like to acknowledge Denise Shortino of PPD for her statistical expertise and assistance with the conduct of this clinical trial. Editorial assistance (graphics recreation, copyediting) was provided by AOIC Communications, Exton, PA, and funded by GlaxoSmithKline.

\section{Funding}

All studies contained in this paper were funded by GlaxoSmithKline, Inc.

\section{Availability of data and materials}

Raw preclinical and clinical data for this manuscript will not be shared for logistical reasons.

\section{Authors' contributions}

SEH and JPK carried out the immunology and transgenic mouse pharmacology studies. AVM and HFK carried out preclinical study design and in situ muscle injury studies. GL carried out preclinical immunoassays on in vivo samples. JMF and DKR participated in bioinformatic analysis of Somalogic and transcriptional 
profiling data. RSG performed histology work. ZC participated in the clinical statistical analysis. ANB, AJR, SEH, and ACH conceived of the preclinical studies. $A J R, J L, A C, R M, R V C, B Y$, and FF conceived and planned the clinical study. KN provided technical direction for eccentric exercise protocols. AJR, ANB, JPK, and $\mathrm{ACH}$ helped to draft the manuscript. All authors read and approved the final manuscript.

\section{Ethics approval and consent to participate}

All studies with mice were conducted in accordance with the GSK Policy on the Care, Welfare and Treatment of Laboratory Animals. All protocols were reviewed and approved by the Institutional Animal Care and Use Committee of GSK.

The clinical study protocol, any amendments, the informed consent, and other information that required pre-approval were reviewed and approved by an investigational center institutional review board, in accordance with the International Conference on Harmonization of Technical Requirements for Registration of Pharmaceuticals for Human Use (ICH) Good Clinical Practice (GCP) and applicable country-specific requirements, including United States (US) 21 Code of Federal Regulations (CFR) 312.3(b) for constitution of independent ethics committees.

The study was conducted in accordance with ICH GCP and all applicable subject privacy requirements, and, the ethical principles that are outlined in the Declaration of Helsinki 2008. The study was monitored in accordance with ICH E6, Section 5.18. Investigators were trained to conduct the study in accordance with GCPs and the study protocol as defined in ICH E3, Section 9.6. Written commitments were obtained from investigators to comply with GCP and to conduct the study in accordance with the protocol. Written informed consent was obtained from each subject prior to the performance of any study-specific procedures. The investigator agreed to provide the subjects as much time as necessary to review the document, to inquire about details of the trial, and to decide whether or not to participate in the study. The informed consent was signed and dated by the study subjects and by the person who conducted the informed consent discussion. Electronic case report forms were provided for each subject's data to be recorded.

\section{Competing interests}

$A B, S H, A V D, G L, A C H, J P K, J M F, Z C, D K R, H F K, R S G, F F, B Y, R C, J L, A C, R M$, and AJR were employees and shareholders of GlaxoSmithKline at the time of study conduct. KN was an employee at Edith Cowan University, Joondalup, Australia, at the time of study conduct.

\section{Publisher's Note}

Springer Nature remains neutral with regard to jurisdictional claims in published maps and institutional affiliations.

\section{Author details}

${ }^{1}$ Muscle Metabolism Discovery Performance Unit, GlaxoSmithKline, King of Prussia, PA, USA. ${ }^{2}$ Metabolic Pathways and Cardiovascular Therapy Area, GlaxoSmithKline, King of Prussia, PA, USA. ${ }^{3}$ Target Sciences, GlaxoSmithKline, King of Prussia, PA, USA. ${ }^{4}$ Clinical Statistics, GlaxoSmithKline, King of Prussia, PA, USA. ${ }^{5}$ School of Medical and Health Sciences, Edith Cowan University, Joondalup, WA, Australia.

\section{Received: 25 May 2018 Accepted: 14 October 2018} Published online: 13 November 2018

\section{References}

1. Carlson BM, Faulkner JA. The regeneration of skeletal muscle fibers following injury: a review. Med Sci Sports Exerc. 1983;15(3):187-98.

2. Semenza GL. Regulation of mammalian $\mathrm{O} 2$ homeostasis by hypoxia-inducible factor 1. Annu Rev Cell Dev Biol. 1999;15:551-78.

3. Mann CJ, Perdiguero E, Kharraz Y, Aguilar S, Pessina P, Serrano AL, et al. Aberrant repair and fibrosis development in skeletal muscle. Skelet Muscle 2011;1(1):21.

4. Tang K, Breen EC, Wagner H, Brutsaert TD, Gassmann M, Wagner PD. HIF and VEGF relationships in response to hypoxia and sciatic nerve stimulation in rat gastrocnemius. Respir Physiol Neurobiol. 2004;144(1):71-80.

5. Gustafsson T, Puntschart A, Kaijser L, Jansson E, Sundberg CJ. Exerciseinduced expression of angiogenesis-related transcription and growth factors in human skeletal muscle. Am J Phys. 1999;276(2 Pt 2):H679-85.
6. Rissanen TT, Vajanto I, Hiltunen MO, Rutanen J, Kettunen MI, Niemi M, et al. Expression of vascular endothelial growth factor and vascular endothelial growth factor receptor-2 (KDR/FIk-1) in ischemic skeletal muscle and its regeneration. Am J Pathol. 2002;160(4):1393-403.

7. Richardson RS, Noyszewski EA, Kendrick KF, Leigh JS, Wagner PD. Myoglobin $\mathrm{O} 2$ desaturation during exercise. Evidence of limited O2 transport. J Clin Invest. 1995;96(4):1916-26.

8. Scheerer N, Dehne N, Stockmann C, Swoboda S, Baba HA, Neugebauer A, et al. Myeloid hypoxia-inducible factor-1alpha is essential for skeletal muscle regeneration in mice. J Immunol. 2013;191(1):407-14.

9. Schofield CJ, Ratcliffe PJ. Oxygen sensing by HIF hydroxylases. Nat Rev Mol Cell Biol. 2004;5(5):343-54.

10. Deng A, Arndt MA, Satriano J, Singh P, Rieg T, Thomson S, et al. Renal protection in chronic kidney disease: hypoxia-inducible factor activation vs. angiotensin II blockade. Am J Physiol Renal Physiol. 2010;299(6):F1365-73.

11. Lindholm ME, Rundqvist $H$. Skeletal muscle hypoxia-inducible factor-1 and exercise. Exp Physiol. 2016;101(1):28-32

12. Faulkner JA, Brooks SV, Opiteck JA. Injury to skeletal muscle fibers during contractions: conditions of occurrence and prevention. Phys Ther. 1993: 73(12):911-21

13. Pratt SJ, Lawlor MW, Shah SB, Lovering RM. An in vivo rodent model of contraction-induced injury in the quadriceps muscle. Injury. 2012;43(6):788-93.

14. Bao W, Qin P, Needle S, Erickson-Miller CL, Duffy KJ, Ariazi JL, et al. Chronic inhibition of hypoxia-inducible factor prolyl 4-hydroxylase improves ventricular performance, remodeling, and vascularity after myocardial infarction in the rat. J Cardiovasc Pharmacol. 2010;56(2):147-55.

15. Jia Y, Suzuki N, Yamamoto M, Gassmann M, Noguchi CT. Endogenous erythropoietin signaling facilitates skeletal muscle repair and recovery following pharmacologically induced damage. FASEB J. 2012;26(7):2847-58.

16. Hardee ME, Cao Y, Fu P, Jiang X, Zhao Y, Rabbani ZN, et al. Erythropoietin blockade inhibits the induction of tumor angiogenesis and progression. PLoS One. 2007;2(6):e549.

17. Kellner A, Robertson T. Selective necrosis of cardiac and skeletal muscle induced experimentally by means of proteolytic enzyme solutions given intravenously. J Exp Med. 1954;99(4):387-404.

18. Gondin J, Theret M, Duhamel G, Pegan K, Mathieu JR, Peyssonnaux C, et al. Myeloid HIFs are dispensable for resolution of inflammation during skeletal muscle regeneration. J Immunol. 2015;194(7):3389-99.

19. Tidball JG, Villalta SA. Regulatory interactions between muscle and the immune system during muscle regeneration. Am J Physiol Regul Integr Comp Physiol. 2010;298(5):R1173-87.

20. Tidball JG, Wehling-Henricks M. Macrophages promote muscle membrane repair and muscle fibre growth and regeneration during modified muscle loading in mice in vivo. J Physiol. 2007;578(Pt 1):327-36.

21. Riboldi E, Porta C, Morlacchi S, Viola A, Mantovani A, Sica A. Hypoxiamediated regulation of macrophage functions in pathophysiology. Int Immunol. 2013;25(2):67-75.

22. Buono R, Vantaggiato C, Pisa V, Azzoni E, Bassi MT, Brunelli S, et al. Nitric oxide sustains long-term skeletal muscle regeneration by regulating fate of satellite cells via signaling pathways requiring Vangl2 and cyclic GMP. Stem Cells. 2012;30(2):197-209.

23. Rigamonti E, Touvier T, Clementi E, Manfredi AA, Brunelli S, Rovere-Querin $P$. Requirement of inducible nitric oxide synthase for skeletal muscle regeneration after acute damage. J Immunol. 2013;190(4):1767-77.

24. Tidball JG, Dorshkind K, Wehling-Henricks M. Shared signaling systems in myeloid cell-mediated muscle regeneration. Development. 2014;141(6): 1184-96.

25. Garvey EP, Oplinger JA, Furfine ES, Kiff RJ, Laszlo F, Whittle BJ, et al. 1400W is a slow, tight binding, and highly selective inhibitor of inducible nitricoxide synthase in vitro and in vivo. J Biol Chem. 1997;272(8):4959-63.

26. Laubach VE, Shesely EG, Smithies O, Sherman PA. Mice lacking inducible nitric oxide synthase are not resistant to lipopolysaccharide-induced death. Proc Natl Acad Sci U S A. 1995;92(23):10688-92.

27. Clarkson PM, Hubal MJ. Exercise-induced muscle damage in humans. Am J Phys Med Rehabil. 2002;81(11 Suppl):S52-69.

28. Johnson BM, Stier BA, Caltabiano S. Effect of food and gemfibrozil on the pharmacokinetics of the novel prolyl hydroxylase inhibitor GSK1278863. Clin Pharmacol Drug Dev. 2014;3(2):109-17.

29. Schneider M, Van Geyte K, Fraisl P, Kiss J, Aragones J, Mazzone M, et al. Loss or silencing of the PHD1 prolyl hydroxylase protects livers of mice against ischemia/reperfusion injury. Gastroenterology. 2010;138(3):1143-54 e1 2. 
30. Chen RL, Nagel S, Papadakis M, Bishop T, Pollard P, Ratcliffe PJ, et al. Roles of individual prolyl-4-hydroxylase isoforms in the first 24 hours following transient focal cerebral ischaemia: insights from genetically modified mice. J Physiol. 2012;590(16):4079-91.

31. Barker JE, Knight KR, Romeo R, Hurley JV, Morrison WA, Stewart AG. Targeted disruption of the nitric oxide synthase 2 gene protects against ischaemia/reperfusion injury to skeletal muscle. J Pathol. 2001;194(1):109-15.

32. Khanna A, Cowled PA, Fitridge RA. Nitric oxide and skeletal muscle reperfusion injury: current controversies (research review). J Surg Res. 2005; 128(1):98-107.

33. Ogle ME, Segar CE, Sridhar S, Botchwey EA. Monocytes and macrophages in tissue repair: implications for immunoregenerative biomaterial design. Exp Biol Med (Maywood). 2016;241(10):1084-97.

34. Ariazi JL, Duffy KJ, Adams DF, Fitch DM, Luo L, Pappalardi M, et al. Discovery and preclinical characterization of GSK1278863 (Daprodustat), a small molecule hypoxia inducible factor-prolyl hydroxylase inhibitor for anemia. J Pharmacol Exp Ther. 2017;363(3):336-47.

35. Escribese MM, Sierra-Filardi E, Nieto C, Samaniego R, Sanchez-Torres C, Matsuyama T, et al. The prolyl hydroxylase PHD3 identifies proinflammatory macrophages and its expression is regulated by activin A.J Immunol. 2012; 189(4):1946-54.

36. Warren GL, Ingalls CP, Lowe DA, Armstrong RB. Excitation-contraction uncoupling: major role in contraction-induced muscle injury. Exerc Sport Sci Rev. 2001;29(2):82-7.

37. Yu JG, Malm C, Thornell LE. Eccentric contractions leading to DOMS do not cause loss of desmin nor fibre necrosis in human muscle. Histochem Cell Biol. 2002;118(1):29-34

38. Yu JG, Carlsson L, Thornell LE. Evidence for myofibril remodeling as opposed to myofibril damage in human muscles with DOMS: an ultrastructural and immunoelectron microscopic study. Histochem Cell Biol. 2004;121(3):219-27.

39. Nosaka K, Clarkson PM, McGuiggin ME, Byrne JM. Time course of muscle adaptation after high force eccentric exercise. Eur J Appl Physiol Occup Physiol. 1991;63(1):70-6.

40. Hathout Y, Brody E, Clemens PR, Cripe L, DeLisle RK, Furlong P, et al. Largescale serum protein biomarker discovery in Duchenne muscular dystrophy. Proc Natl Acad Sci U S A. 2015:112(23):7153-8.

41. Gautier L, Cope L, Bolstad BM, Irizarry RA. affy-analysis of Affymetrix GeneChip data at the probe level. Bioinformatics. 2004;20(3):307-15.

42. Dai M, Wang P, Boyd AD, Kostov G, Athey B, Jones EG, et al. Evolving gene/ transcript definitions significantly alter the interpretation of GeneChip data. Nucleic Acids Res. 2005;33(20):e175-e.

43. Smyth GK. Linear models and empirical bayes methods for assessing differential expression in microarray experiments. Stat Appl Genet Mol Biol. 2004;3:Article3.

44. Ritchie ME, Phipson B, Wu D, Hu Y, Law CW, Shi W, et al. limma powers differential expression analyses for RNA-sequencing and microarray studies. Nucleic Acids Res. 2015:43(7):e47.

45. Benjamini $Y$, Hochberg $Y$. Controlling the false discovery rate: a practical and powerful approach to multiple testing. J R Stat Soc Ser B (Methodological). 1995;57(1):289-300.

46. Huber W, Carey VJ, Gentleman R, Anders S, Carlson M, Carvalho BS, et al. Orchestrating high-throughput genomic analysis with Bioconductor. Nat Methods. 2015;12(2):115-21.

Ready to submit your research? Choose BMC and benefit from:

- fast, convenient online submission

- thorough peer review by experienced researchers in your field

- rapid publication on acceptance

- support for research data, including large and complex data types

- gold Open Access which fosters wider collaboration and increased citations

- maximum visibility for your research: over $100 \mathrm{M}$ website views per year

At $\mathrm{BMC}$, research is always in progress.

Learn more biomedcentral.com/submissions 